\title{
Considerations for immunotherapy in patients with cancer and comorbid immune dysfunction
}

\author{
Vaia Florou $^{1}$, Sonam Puri ${ }^{1}$, Ignacio Garrido-Laguna ${ }^{1}$, Breelyn A. Wilky ${ }^{2}$ \\ ${ }^{1}$ Department of Internal Medicine, Division of Oncology, Huntsman Cancer Institute, University of Utah School of Medicine, Salt Lake City, UT, \\ USA; ${ }^{2}$ Department of Medicine, Division of Medical Oncology, University of Colorado School of Medicine, Aurora, CO, USA \\ Contributions: (I) Conception and design: V Florou; (II) Administrative support: None; (III) Provision of study material or patients: None; (IV) \\ Collection and assembly of data: None; (V) Data analysis and interpretation: None; (VI) Manuscript writing: All authors; (VII) Final approval of \\ manuscript: All authors. \\ Correspondence to: Vaia Florou, MD. Department of Internal Medicine, Division of Oncology, Huntsman Cancer Institute, University of Utah School \\ of Medicine, 2000 Circle of Hope, Salt Lake City, UT, USA. Email: vaia.florou@hci.utah.edu.
}

\begin{abstract}
Immune checkpoint inhibitors have been widely incorporated for cancer treatment in a variety of solid and hematologic malignancies. Multiple clinical trials have demonstrated the efficacy of PD-1/PD-L1 and CTLA-4 axis inhibition in the metastatic and adjuvant settings. Due to the risks of autoimmune toxicity with these agents, stringent inclusion/exclusion criteria were employed in those initial clinical trials. These criteria led to exclusion or underrepresentation of a variety of patient populations with underlying immune dysfunction. These populations included patients with preexisting autoimmune diseases, solid organ or bone marrow transplant recipients, patients with HIV or viral hepatitis infections, patients receiving concurrent chronic steroid therapy, as well as patients who were elderly, pregnant, or had poor performance status. Thus, established guidelines on the use of immune checkpoint inhibitors in these patients are lacking, and evidence to support efficacy or toxicity are overall limited to retrospective studies and case series. Fortunately, ongoing clinical trials are now including these patients and are shedding light on whether these underrepresented populations can also safely benefit from immune checkpoint inhibitor therapies. In this review, we summarize the most clinically relevant available data on the use of checkpoint inhibitors in immunocompromised patient groups with a primary focus on safety.
\end{abstract}

Keywords: Immunotherapy; checkpoint inhibitors; immunosuppressed patients

Submitted Jul 08, 2020. Accepted for publication Oct 28, 2020.

doi: $10.21037 / \mathrm{atm}-20-5207$

View this article at: http://dx.doi.org/10.21037/atm-20-5207

\section{Introduction}

It has been almost a decade since immune evasion was recognized as a hallmark of cancer (1), and since then, cancer immunotherapy has been well integrated into the treatment of numerous solid and hematological malignancies. One of the most common cancer immunotherapy applications relates to the use of checkpoint inhibitors, which are monoclonal antibodies that block immune suppression mediated by PD-1/PD-L1 and CTLA-4 signaling. An array of agents is already approved by the Food and Drug Administration (FDA) and an even larger number of checkpoint inhibitors are currently in preclinical and clinical studies. Immunotherapy, contrary to cytotoxic chemotherapy, has a completely different mechanism of action and aims to disrupt the symbiosis between the immune system and cancer. A key step in the process of the cancer immunoediting shift, which eventually leads to tumor growth and proliferation, is mediated by the escape

^ ORCID: 0000-0001-5558-753X. 
of the tumor cells from the equilibrium state where tumor dormancy occurs with no apparent clinical disease (2). While these mechanisms are complex and not yet fully understood, what has become clear is that immunosuppressed patients do have a higher incidence of certain malignancies $(3,4)$. By reversing immunosuppression with immunotherapy agents, immune attack against tumor can be restored, but often results in autoimmunity against normal cells. In patients with underlying immune dysfunction, including those with iatrogenic or acquired immunosuppression or preexisting autoimmune conditions, the risks of further disruption of the immune system's checks and balances with checkpoint inhibitors could outweigh potential anti-cancer benefits. However, limited data exist to date for whether this theory holds true in this unique population of patients. This review aims to provide an overview of the available data on immunotherapy applications in immunosuppressed patients.

\section{Organ transplant recipients}

Solid-organ and allogeneic hematopoietic stem cell transplant (HSCT) recipients must remain on chronic immunosuppression to maintain graft tolerance and prevent graft-versus-host disease (GVHD). GVHD most commonly occurs with stem cell transplantation, although it can occur with any transplanted graft which contains large amount of donor immune cells (5).

Graft rejection and GVHD are two major immunological complications of HSCT mediated by a complex crosstalk among predominantly $\mathrm{T}$ cells, innate lymphoid cells, intestinal epithelial cells, microbiota and stromal cells in secondary lymphoid organs (5). In allogenic HSCT, PDL1 expression on donor T-cells is functionally implicated in regulating acute GVHD suggesting that inhibition of the axis may prevent GVHD (6). To investigate this, a phase 1 trial on the single-agent CTLA-4 inhibitor, ipilimumab, dosed at $0.1-3 \mathrm{mg} / \mathrm{kg}$, for patients with relapsed hematologic malignancies after allogeneic HSCT, included 29 patients, none of whom developed any grade 3 or 4 acute GVHD. Still, four patients developed distinct irAEs (immune-related adverse events) (7), of which one was grade 3 polyarthropathy and one grade 4 pneumonitis.

In contrast, when the same agent used at higher doses of 3 or $10 \mathrm{mg} / \mathrm{m}^{2}$ in a subsequent study of 28 patients, GVHD rates were $14 \%$, and six patients $(21 \%)$ experienced irAEs, with two $\geq$ grade 3 toxicities and one treatment-related death (8). PD-1 inhibition has been used successfully in numerous case reports of patients with recurrent hematologic malignancies after HSCT (9-14) without major GVHD complications. Looking at larger retrospective studies, though, various degrees of GVHD have been reported. In a 30-patient study of patients who received anti-PD-1 therapy post allogeneic HSCT, the response rate was $77 \%$; however, the GVHD rate was as high as $55 \%$ (grade 3-4 acute or severe chronic; 9 patients) with eight deaths related to new-onset GVHD (15). Fatal GVHD has also been reported with pembrolizumab after allogeneic GVHD (16). An interesting question raised in some successfully treated cases is whether lower doses of PD-1 antibody can mitigate the risk of GVHD and development of irAEs while maintaining efficacy.

On the same note, the evidence is also controversial on the safety of checkpoint inhibition in solid organ transplant recipients. The immunosuppressive state required for allograft tolerance, increases the risk of de novo malignancies and complicates the therapy of these secondary cancers. Preclinical evidence suggest that the PD-1/PD-L1 axis is important for solid organ allograft tolerance $(17,18)$. In a 35-patient cohort study of solid transplant patients treated for second malignancies with immunotherapy, the overall response rate (ORR) of immune checkpoint inhibitors was $43.4 \%$, and graft rejection rate was $31.4 \%$ in patients with liver, renal, or heart transplants (19). A systematic review of transplant patients receiving either PD-1 or CTLA-4 inhibitors reported the highest rejection rate in patients with kidney $(40.1 \%)$, followed by liver (35\%) and heart $(20 \%)$ transplant (20). However, most of the patients included in that metanalysis received renal $(n=32)$ transplants followed by liver transplants $(n=20)$ and only 5 received heart transplants (20). Interestingly, there was no association between the particular checkpoint inhibitor used and rejection rates, with most patient deaths ultimately attributed to disease progression rather than graft rejection (20). An analysis of exclusively renal transplant recipients receiving checkpoint inhibitors included 44 patients, 18 of whom had graft rejection, and 10 of them eventually died of various etiologies unrelated to irAEs (21). Overall, the advent of potent immunosuppressive therapy has led to a decreasing incidence of kidney acute rejection in the first year post transplant, which has been consistently less than $10 \%$ (22). Whether the degree of immunosuppression plays a role in this high graft failure in solid transplants with checkpoint inhibition or the actual transplanted organ is unclear. Although the data are limited thus far and derived mainly from retrospective case series and case reports, risks and benefits of this approach in this context should be carefully weighed considering the 
type of the transplanted organ and the expected efficacy of checkpoint inhibitors.

\section{Autoimmune disorders}

Patients with pre-existing autoimmune disorders present a major common clinical challenge because of the immunosuppressive treatment often required and due to the inherent impairment of the immune system leading to those conditions. There is also the concern of worsening or exacerbating the underlying autoimmune disorder especially those driven mainly by $\mathrm{T}$ cells i.e., rheumatoid arthritis or multiple sclerosis. Although the precise pathophysiology mediating irAEs is not fully understood and there is a lack of predictive factors, pre-existing dysregulation of the immune system raises the concern of potentially impacting the severity, incidence and timing of irAEs with checkpoint inhibitors. Since these patients were initially almost universally excluded from the immunotherapy trials, most evidence is based on retrospective studies and case reports reported in the literature. A recent systematic analysis included 123 patients with a variety of autoimmune disorders - psoriasis, rheumatoid arthritis, autoimmune thyroid disease, ulcerative colitis, Crohn disease, multiple sclerosis, myasthenia gravis, and sarcoidosis (23). The majority of those patients, $83.5 \%$, received prior treatment of their autoimmune disease, $46.5 \%$ had an active autoimmune disease, and $43.6 \%$ were receiving concurrent immunosuppression at the time of immunotherapy initiation. Overall, $41 \%$ of patients had an exacerbation of their pre-existing autoimmune disease with a similar presentation of their original disease, $25 \%$ had de novo irAEs, and $17.1 \%$ of all patients had to discontinue therapy due to irAEs (23). Half the patients who developed irAEs had either partial or complete response vs. $35.7 \%$ of patients who did not experience any irAEs (23). Interestingly there was no difference in the frequency of irAEs between patients with prior active autoimmune disorders versus inactive, and patients who were receiving immunosuppressive treatment concomitantly with immunotherapy had actually fewer irAEs (59\% vs. $83 \%)(23)$. Importantly, in more than half of the patients, there was no need to discontinue immunotherapy. Another interesting point of this analysis is the observation that antiCTLA-4 agents were associated with more de novo irAEs compared to PD-1/PD-L1 axis inhibitors ( $42 \%$ vs. $26 \%$, respectively), which were associated more with the patients' pre-existing autoimmune disease flares (23). A large multicenter French retrospective study on a similar patient population included 112 patients and reported similar results-overall, irAEs developed in $71 \%$ of patients, with $47 \%$ experiencing a flare of their autoimmune disorder, $42 \%$ developing de novo irAEs, and $18 \%$ developing both (24). Only $21 \%$ of patients discontinued their treatment because of irAEs (24). This study also reported a key observation that immunosuppressive treatment at the time of immunotherapy did confer a negative impact on cancer survival; shorter progression-free survival [HR 2.10 (95\% CI: $1.08-4.07$ ), $\mathrm{P}=0.028$ ] and a trend towards worse overall survival although not statistically significant [HR 1.95 (95\% CI: 0.89-4.64), P=0.134] (24). Lastly, in another recent retrospective study which included 46 patients with prior autoimmune conditions (hypothyroidism, psoriasis, rheumatoid arthritis, lupus, myasthenia gravis, inflammatory bowel disease and others) nine patients had flares with checkpoint inhibition, and only one patient required discontinuation of immunotherapy (25).

Considering all these data, pre-existing autoimmune disorders should not necessarily be an absolute contraindication, especially when the potential anti-cancer benefits outweigh the risks. While there is a potential of reactivation of the autoimmune disorder, this is not universal, and more importantly, few patients required therapy discontinuation despite the development of irAEs or disease flare. Future research on the pathogenesis of irAEs will hopefully improve the ability to predict irAEs and guide the use of checkpoint inhibitors minimizing toxicity.

Lastly, a unique condition in this population is the cancer-associated dermatomyositis which can be present either at the time or years prior the diagnosis of cancer. This is a challenging disease and it represents a good example of the complex relationship between cancer and autoimmunity. Interestingly, a study recently showed that patients with dermatomyositis and cancer had significantly higher levels of soluble PD-L1 expression when compared to patients without cancer or those with cancer in remission (26). While causation cannot be elicited from this early study, it can be hypothesized that patients with cancer and dermatomyositis may have higher responses to immune checkpoint inhibitors.

\section{Acquired immunosuppression-viral infections or chronic steroid use}

Another challenging population is the patients who have acquired immunosuppression secondary to either viral 
infections like HIV or chronic steroid use. Importantly, the patients with concomitant HIV or hepatitis infections were all excluded from the first clinical trials with checkpoint inhibitors, due to the viral immunosuppressive effects on $\mathrm{T}$ cells. Independently from the coinfection with HIV, human hepatitis viruses and in particular HCV activate innate immune responses and can lead to $\mathrm{T}$ cell exhaustion due to chronic antigen stimulation (27). This prolonged activation of the innate immunity which can potentially impair adaptive immune responses, may ultimately confer vulnerability to checkpoint inhibition. Limited data suggest the beneficial effects of checkpoint inhibitors not only against cancer but also on HIV viral clearance and increase of CD4, and CD8 T lymphocyte counts $(28,29)$. More recent retrospective series reported similar findings; a 50-patient study, which included both patients with HIV and viral hepatitis, showed an ORR to checkpoint inhibition of $28 \%$ and $18 \%$, respectively (30). The incidence of any grade irAEs was $24 \%$ and $44 \%$ in the HIV and the HBV/ HCV cohorts, respectively (30). A systematic review of 73 patients with HIV infection reported even better tolerance to checkpoint inhibitors with an overall irAE rate of $8.6 \%$ and ORR of $30 \%$ for lung cancer, $27 \%$ for melanoma, and $63 \%$ for Kaposi sarcoma (31). Patients with HIV and viral hepatitis infections appear to have similar tolerance to checkpoint inhibitors and in some malignancies possibly enhanced antitumor efficacy—with the most recent example of superior overall survival of patients with viral associated hepatocellular carcinoma when treated with Bevacizumab in combination with checkpoint inhibition compared to those with non-viral associated disease (32). Multiple trials in the last few years with checkpoint inhibitors are now including patients with HIV infections to answer these questions prospectively.

On the other hand, patients on chronic steroid use for multiple reasons beyond autoimmune disorders constitute another very common and challenging clinical scenario. There is accumulating evidence that chronic steroid use may harm immunotherapy responses depending on the dose and the timing of initiation. In the first study on a cohort of 640 patients with metastatic lung cancer and concurrent use of prednisone, dose $\geq 10 \mathrm{mg}$ /day was associated with lower response rates and survival compared to those patients who were using prednisone at $<10 \mathrm{mg} /$ day (33). These findings were consistent with the results of several other retrospective studies, especially when steroid exposure was early during immunotherapy (34). Similarly, one of the first trials of CTLA-4 inhibition in patients with melanoma and brain metastases included patients who were on stable corticosteroid dose and showed worse survival compared to those patients who were off steroids at the time of treatment (35). Although these conclusions are mainly based on retrospective studies, they do offer a plausible hypothesis of why steroid use does not affect efficacy when given later for the treatment of irAEs (36) when T cell antitumor response is already established. Steroids affect $\mathrm{T}$ cell apoptosis (37), and induce $\mathrm{T}$ regulatory cell proliferation and recruitment (38), which can eventually lead to primary or adaptive resistance to checkpoint inhibitors.

\section{Other immunosuppressed populations- pregnancy and the elderly}

In contrast to the populations mentioned above, some patients represent a challenge due to functional baseline immunosuppression resulting from a physiologic state such as pregnancy or advanced age.

Checkpoint regulators like PD-1 and CTLA-4 play an important role in maintaining maternal-fetal immunotolerance in pregnancy (39). Anti-PD-1 and antiCTLA-4 antibodies are categorized as pregnancy category $\mathrm{D}$ and $\mathrm{C}$, respectively, by the FDA. While scarce case reports in the literature of patients who were found to be pregnant while on checkpoint inhibitors $(40,41)$ or were treated with checkpoint inhibition during gestation (42) reported favorable pregnancy and oncologic outcomes, no prospective or large retrospective studies are available. Hence, treatment decisions in this setting should be highly individualized based on the potential benefits and risks for the patient and fetal safety.

Most elderly patients were excluded from immunotherapy trials as well, and information on the interactions of checkpoint inhibition and aged immune cells is limited. However, as the population older than 80 years in oncology is increasing, this is slowly becoming a challenge in clinical practice. While aging is associated with a decline in immune function, elderly are not considered strictly immunodeficient (43). The response to antigens with aging is reduced, and thus it can possibly affect the immune tumor microenvironment $(43,44)$. However, clinically this impact has not been studied, and the functional status is more of consideration rather than patient's biologic age when checkpoint inhibitors are prescribed. A large metaanalysis of 5,265 patients, which dichotomized patients into younger and older groups with an age cutoff of $65-70$, showed a consistent benefit of immunotherapy in both age 
groups (45). Similar findings were reported in a metaanalysis of patients with lung cancer treated with checkpoint inhibitors when the cutoff of 65 years was used (46). Responses to checkpoint inhibition have been reported even in patients more than 90 years old with acceptable tolerance (47). Ultimately, in the absence of other contraindications, the performance status and remaining comorbidities should carry a higher impact on clinical decision making. Close monitoring and timely management of adverse events are crucial for this population to ensure the safety of checkpoint inhibitors.

\section{Poor performance status}

While there is strong evidence that the administration of cytotoxic chemotherapy in patients with poor performance status is associated with worse toxicity which overcomes potential efficacy, the impact of performance status on the safety and efficacy of immunotherapy is unclear. Since the side effect profile of checkpoint inhibitors is drastically different than that of chemotherapy, immunotherapy may be an appealing option in patients whose only option would be the best supportive care. There are very few trials that included patients with an ECOG performance status of 2 (48-52), mainly of patients with lung or urothelial cancer. Most of these trials included a mix of elderly patients and performance status $0-2$. The clinical benefit appeared to be consistent regardless of poor performance status with similar toxicity rates to that of patients with a performance status of $0-1$ (48-52). A meta-analysis of 18 studies analyzed 11,354 patients who received immunotherapy for solid tumors did not show any significant difference between patients with a performance status of 0 versus $1-2$ (53).

In contrast, a more recent meta-analysis of 3,600 patients with exclusively lung cancer, reported that patients with performance status $\geq 2$ had overall inferior survival outcomes (54). This finding, however, could be confounded by multiple factors such as more aggressive disease biology and patient heterogeneity, not necessarily reflecting the lower efficacy of immunotherapy in this setting (54). Other trials specifically for patients with a performance status 2-3 are currently ongoing (e.g., NCT04221529, NCT02879617, NCT04108026).

Despite the data heterogeneity, in carefully selected patients with poor performance status regardless of age, checkpoint inhibition may be very efficient and lead to prolongation of life; the histology, tumor characteristics, and biomarkers of response such as PD-L1 expression and tumor mutation burden in certain malignancies should also be accounted for prior making the treatment decision.

\section{Conclusions}

Immunotherapy has been undoubtedly a breakthrough in cancer treatment and completely altered the treatment landscape of multiple malignancies. The side effect profile of immunotherapy can be similar to autoimmune disorders creating concerns of higher toxicity rates and decreased efficacy in patients with underlying autoimmune diseases and/or immunosuppression. Hence, this population was excluded from the majority of the initial clinical trials, and available data on the safety and efficacy of immune checkpoint inhibitors in immunosuppressed populations are mainly from retrospective studies and case series. Despite this, with the increased risk of cancers arising in immunosuppressed patients, there is an unmet need to expand the treatment landscape in this patient population.

As more literature becomes available and more trials are including those patients, this ambiguity will become less prevalent. For most immunosuppressed patient groups, checkpoint inhibitors appear safe or at least not more toxic than those without any underlying immunosuppression. In addition, strategies to mitigate the development of irAEs and to understand the exact mechanisms involved are currently under study and may be particularly relevant for this high-risk population if proven efficacious. A clinical trial of combining dual checkpoint inhibitors with CD24Fc in an effort suppress the danger-associated molecular patterns by binding to Siglec10 (NCT04060407) and decrease irAEs is currently ongoing. As far as efficacy, concurrent immunosuppression may ultimately have an adverse effect. In the metastatic setting, especially when no other therapies are available, even a modest efficacy may be clinically relevant when compared to best supportive treatment. What is important to emphasize is that these patients overall should not be automatically excluded from these treatments that could potentially benefit them. The caveat is, of course, that for specific groups such as pregnancy and vital organ transplant recipients, our knowledge is extremely limited, and the decision should always be made on an individualized basis with a multidisciplinary approach. As the checkpoint inhibitors move to earlier lines of therapy and/or maintenance, one must consider all those risks and carefully weigh them against the potential merits as the risk-benefit ratio in this setting may not be as favorable for this patient population. 
Lastly, biomarkers to predict responses to immunotherapy and limit toxicities need further investigation for all cancer patients but would certainly be helpful to weigh the risks and benefits for high-risk populations.

\section{Acknowledgments}

Funding: None.

\section{Footnote}

Provenance and Peer Review: This article was commissioned by the Guest Editors (Umang Swami and Mohammed M. Milhem) for the series "Cancer Immunotherapy: Recent Advances and Challenges" published in Annals of Translational Medicine. The article has undergone external peer review.

Conflicts of Interest: All authors have completed the ICMJE uniform disclosure form (available at http:// dx.doi.org/10.21037/atm-20-5207). The series "Cancer Immunotherapy: Recent Advances and Challenges" was commissioned by the editorial office without any funding or sponsorship. SP reports other from AstraZeneca, other from G1 therapeutics, outside the submitted work. IGL reports that he serves as PI in a number of clinical trials for which his institution receives research funding. The authors have no other conflicts of interest to declare.

Ethical Statement: The authors are accountable for all aspects of the work in ensuring that questions related to the accuracy or integrity of any part of the work are appropriately investigated and resolved.

Open Access Statement: This is an Open Access article distributed in accordance with the Creative Commons Attribution-NonCommercial-NoDerivs 4.0 International License (CC BY-NC-ND 4.0), which permits the noncommercial replication and distribution of the article with the strict proviso that no changes or edits are made and the original work is properly cited (including links to both the formal publication through the relevant DOI and the license). See: https://creativecommons.org/licenses/by-nc-nd/4.0/.

\section{References}

1. Hanahan D, Weinberg RA. Hallmarks of cancer: The next generation. Cell 2011;144:646-74.

2. Schreiber RD, Old LJ, Smyth MJ. Cancer Immunoediting: Integrating Immunity's Roles in Cancer Suppression and Promotion. Science 2011;331:1565-70.

3. Grulich AE, van Leeuwen MT, Falster MO, et al. Incidence of cancers in people with HIV/AIDS compared with immunosuppressed transplant recipients: a metaanalysis. Lancet 2007;370:59-67.

4. Giat E, Ehrenfeld M, Shoenfeld Y. Cancer and autoimmune diseases. Autoimmun Rev 2017;16:1049-57.

5. Perkey E, Maillard I. New Insights into Graft-VersusHost Disease and Graft Rejection. Annu Rev Pathol 2018;13:219-45.

6. Saha A, O'Connor RS, Thangavelu G, et al. Programmed death ligand-1 expression on donor T cells drives graft-versus-host disease lethality. J Clin Invest 2016;126:2642-60.

7. Bashey A, Medina B, Corringham S, et al. CTLA4 blockade with ipilimumab to treat relapse of malignancy after allogeneic hematopoietic cell transplantation. Blood 2009;113:1581-8.

8. Davids MS, Kim HT, Bachireddy P, et al. Ipilimumab for Patients with Relapse after Allogeneic Transplantation. N Engl J Med 2016;375:143-53.

9. Shad AT, Huo JS, Darcy C, et al. Tolerance and effectiveness of nivolumab after pediatric T-cell replete, haploidentical, bone marrow transplantation: A case report. Pediatr Blood Cancer 2017;64:e26257.

10. Yared JA, Hardy N, Singh Z, et al. Major clinical response to nivolumab in relapsed/refractory Hodgkin lymphoma after allogeneic stem cell transplantation. Bone Marrow Transplant 2016;51:850-2.

11. Angenendt L, Schliemann C, Lutz M, et al. Nivolumab in a patient with refractory Hodgkin's lymphoma after allogeneic stem cell transplantation. Bone Marrow Transplant 2016;51:443-5.

12. Villasboas JC, Ansell SM, Witzig TE. Targeting the PD-1 pathway in patients with relapsed classic Hodgkin lymphoma following allogeneic stem cell transplant is safe and effective. Oncotarget 2016;7:13260-4.

13. Onizuka M, Kojima M, Matsui K, et al. Successful treatment with low-dose nivolumab in refractory Hodgkin lymphoma after allogeneic stem cell transplantation. Int J Hematol 2017;106:141-5.

14. Albring JC, Inselmann S, Sauer T, et al. PD-1 checkpoint blockade in patients with relapsed AML after allogeneic stem cell transplantation. Bone Marrow Transplant 2017;52:317-20. 
15. Haverkos BM, Abbott D, Hamadani M, et al. PD-1 blockade for relapsed lymphoma post-allogeneic hematopoietic cell transplant: high response rate but frequent GVHD. Blood 2017;130:221-8.

16. Singh AK, Porrata LF, Aljitawi O, et al. Fatal GvHD induced by PD-1 inhibitor pembrolizumab in a patient with Hodgkin's lymphoma. Bone Marrow Transplant 2016;51:1268-70.

17. Shi XL, Mancham S, Hansen BE, et al. Counterregulation of rejection activity against human liver grafts by donor PD-L1 and recipient PD-1 interaction. J Hepatol 2016;64:1274-82.

18. Forconi C, Gatault P, Miquelestorena-Standley E, et al. Polymorphism in programmed cell death 1 gene is strongly associated with lung and kidney allograft survival in recipients from CMV-positive donors. J Heart Lung Transplant 2017;36:315-24.

19. DeLeon TT, Salomao MA, Aqel BA, et al. Pilot evaluation of PD-1 inhibition in metastatic cancer patients with a history of liver transplantation: the Mayo Clinic experience. J Gastrointest Oncol 2018;9:1054-62.

20. Fisher J, Zeitouni N, Fan W, et al. Immune checkpoint inhibitor therapy in solid organ transplant recipients: a patient-centered systematic review. J Am Acad Dermatol 2020;82:1490-500.

21. Manohar S, Thongprayoon C, Cheungpasitporn W, et al. Systematic review of the safety of immune checkpoint inhibitors among kidney transplant patients. Kidney Int Rep 2019;5:149-58.

22. Hart A, Smith JM, Skeans MA, et al. OPTN/SRTR 2017 Annual Data Report: Kidney. Am J Transplant 2019;19:19-123.

23. Abdel-Wahab N, Shah M, Lopez-Olivo MA, et al. Use of immune checkpoint inhibitors in the treatment of patients with cancer and preexisting autoimmune disease. Ann Intern Med 2018;169:133.

24. Tison A, Quéré G, Misery L, et al. Safety and efficacy of immune checkpoint inhibitors in patients with cancer and preexisting autoimmune disease: a nationwide, multicenter cohort study. Arthritis Rheumatol 2019;71:2100-11.

25. Kaur A, Doberstein T, Amberker RR, et al. Immunerelated adverse events in cancer patients treated with immune checkpoint inhibitors. Medicine (Baltimore) 2019;98:e17348.

26. Chen H, Peng Q, Yang H, et al. Increased Levels of Soluble Programmed Death Ligand 1 Associate with Malignancy in Patients with Dermatomyositis. J Rheumatol 2018;45:835-40.
27. Park S-H, Rehermann B. Immune Responses to HCV and Other Hepatitis Viruses. Immunity 2014;40:13-24.

28. Galanina N, Goodman AM, Cohen PR, et al. Successful Treatment of HIV-Associated Kaposi Sarcoma with Immune Checkpoint Blockade. Cancer Immunol Res 2018;6:1129-35.

29. Husnain M, Park W, Ramos JC, et al. Complete response to ipilimumab and nivolumab therapy in a patient with extensive extrapulmonary high-grade small cell carcinoma of the pancreas and HIV infection. J Immunother Cancer 2018;6:66.

30. Shah NJ, Al-Shbool G, Blackburn M, et al. Safety and efficacy of immune checkpoint inhibitors (ICIs) in cancer patients with HIV, hepatitis B, or hepatitis $\mathrm{C}$ viral infection. J Immunother Cancer 2019;7:353.

31. Cook MR, Kim C. Safety and efficacy of immune checkpoint inhibitor therapy in patients with HIV infection and advanced-stage cancer. JAMA Oncol 2019;5:1049.

32. Finn RS, Qin S, Ikeda M, et al. Atezolizumab plus Bevacizumab in Unresectable Hepatocellular Carcinoma. N Engl J Med 2020;382:1894-905.

33. Arbour KC, Mezquita L, Long N, et al. Impact of baseline steroids on efficacy of programmed cell death-1 and programmed death-ligand 1 blockade in patients with nonsmall-cell lung cancer. J Clin Oncol 2018;36:2872-8.

34. Rossi G, Pezzuto A, Sini C, et al. Concomitant medications during immune checkpoint blockage in cancer patients: Novel insights in this emerging clinical scenario. Crit Rev Oncol Hematol 2019;142:26-34.

35. Margolin K, Ernstoff MS, Hamid O, et al. Ipilimumab in patients with melanoma and brain metastases: an openlabel, phase 2 trial. Lancet Oncol 2012;13:459-65.

36. Fujii T, Colen RR, Bilen MA, et al. Incidence of immunerelated adverse events and its association with treatment outcomes: the MD Anderson Cancer Center experience. Invest New Drugs 2018;36:638-46.

37. Herold MJ, McPherson KG, Reichardt HM. Glucocorticoids in T cell apoptosis and function. Cell Mol Life Sci 2006;63:60-72.

38. Libert C, Dejager L. How Steroids Steer T Cells. Cell Rep 2014;7:938-9.

39. Miko E, Meggyes M, Doba K, et al. Immune checkpoint molecules in reproductive immunology. Front Immunol 2019; 10:846.

40. Xu W, Moor RJ, Walpole ET, et al. Pregnancy with successful foetal and maternal outcome in a melanoma patient treated with nivolumab in the first trimester. 
Melanoma Res 2019 Jun;29:333-7.

41. Bucheit AD, Hardy JT, Szender JB, et al. Conception and viable twin pregnancy in a patient with metastatic melanoma while treated with CTLA-4 and PD-1 checkpoint inhibition. 2020;30:423-5.

42. Burotto M, Gormaz JG, Samtani S, et al. Viable Pregnancy in a patient with metastatic melanoma treated with double checkpoint immunotherapy. Semin Oncol 2018;45:164-9.

43. Montecino-Rodriguez E, Berent-Maoz B, Dorshkind K. Causes, consequences, and reversal of immune system aging. J Clin Invest 2013;123:958-65.

44. Kaur A, Webster MR, Marchbank K, et al. sFRP2 in the aged microenvironment drives melanoma metastasis and therapy resistance. Nature 2016;532:250-4.

45. Nishijima TF, Muss HB, Shachar SS, et al. Comparison of efficacy of immune checkpoint inhibitors (ICIs) between younger and older patients: A systematic review and metaanalysis. Cancer Treat Rev 2016;45:30-7.

46. Lee CK, Man J, Lord S, et al. Clinical and Molecular Characteristics Associated With Survival Among Patients Treated With Checkpoint Inhibitors for Advanced NonSmall Cell Lung Carcinoma. JAMA Oncol 2018;4:210.

47. Johnpulle RAN, Conry RM, Sosman JA, et al. Responses to immune checkpoint inhibitors in nonagenarians. Oncoimmunology 2016;5:e1234572.

48. Felip E, Ardizzoni A, Ciuleanu T, et al. CheckMate 171: A phase 2 trial of nivolumab in patients with previously treated advanced squamous non-small cell lung cancer, including ECOG PS 2 and elderly populations. Eur J Cancer 2020;127:160-72.

Cite this article as: Florou V, Puri S, Garrido-Laguna I, Wilky BA. Considerations for immunotherapy in patients with cancer and comorbid immune dysfunction. Ann Transl Med 2021;9(12):1035. doi: 10.21037/atm-20-5207
49. Middleton G, Brock K, Savage J, et al. Pembrolizumab in patients with non-small-cell lung cancer of performance status 2 (PePS2): a single arm, phase 2 trial. Lancet Respir Med 2020;8:895-904.

50. Spigel DR, McCleod M, Jotte RM, et al. Safety, Efficacy, and Patient-Reported Health-Related Quality of Life and Symptom Burden with Nivolumab in Patients with Advanced Non-Small Cell Lung Cancer, Including Patients Aged 70 Years or Older or with Poor Performance Status (CheckMate 153). J Thorac Oncol 2019;14:1628-39.

51. Barlesi F, Audigier-Valette C, Felip E, et al. OA04.02 CheckMate 817: First-Line Nivolumab + Ipilimumab in Patients with ECOG PS 2 and Other Special Populations with Advanced NSCLC. J Thorac Oncol 2019;14:S214-5.

52. Grivas P, Plimack ER, Balar AV, et al. Pembrolizumab as First-line Therapy in Cisplatin-ineligible Advanced Urothelial Cancer (KEYNOTE-052): Outcomes in Older Patients by Age and Performance Status. Eur Urol Oncol 2020;3:351-9.

53. Bersanelli M, Brighenti M, Buti S, et al. Patient performance status and cancer immunotherapy efficacy: a meta-analysis. Med Oncol 2018;35:132.

54. Dall'Olio FG, Maggio I, Massucci M, et al. ECOG performance status $\geq 2$ as a prognostic factor in patients with advanced non-small cell lung cancer treated with immune checkpoint inhibitors-A systematic review and meta-analysis of real world data. Lung Cancer 2020;145:95-104. 